\title{
Two Large Open-Access Datasets for Fitts' Law of Human Motion and a Succinct Derivation of the Square-Root Variant
}

\author{
Ken Goldberg $^{\mathrm{a} *}$, Siamak Faridani ${ }^{\mathrm{b}}$ and Ron Alterovitz ${ }^{\mathrm{c}}$ \\ ${ }^{a}$ Professor, UC Berkeley and UC San Francisco, CA, goldberg@berkeley.edu; \\ ${ }^{\mathrm{b}}$ Researcher, Microsoft Silicon Valley, CA, siamakf@microsoft.com ${ }^{\mathrm{c}}$ Assistant Professor, University of North \\ Carolina at Chapel Hill, NC, ron@cs.unc.edu;
}

\begin{abstract}
Human motion models for reaching and pointing tasks are used by designers of software, cellphones, dashboards, cockpits, assembly lines and many other human interfaces. Fitts' Law specifies a logarithmic two-parameter relationship between motion duration and the ratio of target distance over target size. This paper presents two large open-access datasets from experimental user studies, first a controlled (in-lab) study that collected 16,170 trajectory time measurements from 46 participants, and second an uncontrolled (web-based) study that collected 78,410 trajectory time measurements from an uncertain number of participants who used a web-based java applet. Variants of Fitts' Law use square root and modified logarithmic functions; we present a succinct derivation of the square-root variant based on optimal control theory. Using root-mean-square error (RMSE), we compare three two-parameter models that relate motion duration to the ratio of target distance over target size: LOG (Fitts' original logarithmic function), SQR (squareroot), and LOG' (McKenzie's logarithmic plus 1.0) [1]. We find: (1) the data from the controlled and uncontrolled studies are remarkably consistent; (2) for homogeneous targets (with fixed size and distance), the SQR model yields a significantly better fit than LOG or LOG', except with the most difficult targets (where the ratio of target distance over target size is large) where the models are not significantly different; and (3) for heterogeneous targets (with varying size and distance), SQR yields a significantly better fit than LOG for easy targets and LOG yields a significantly better fit for targets of medium difficulty, while the LOG' model yields a significantly better fit than both LOG and SQR on very difficult targets. The anonymized datasets of 94,580 human reaching motion timing measurements are, to our knowledge, the largest collected to date and are freely available at: http://automation.berkeley.edu/fitts/

Index Terms-Fitts' law; human-computer interfaces; time and motion studies; human movement time
\end{abstract}

\section{INTRODUCTION}

The design of almost all human interfaces, from computers to cellphones to games to assembly lines, can benefit from intuitive mathematical models of human motion and ergonomics. The tradeoff between speed and accuracy in human reaching movements was studied by Paul Fitts of Ohio State University in 1954 [2]. Fitts considered reaching movements between rectangular targets, which arise in industrial settings for tasks ranging from installing parts on an assembly line to stamping envelopes in an office. In a series of human studies, subjects

\footnotetext{
${ }^{*}$ Corresponding author.
}

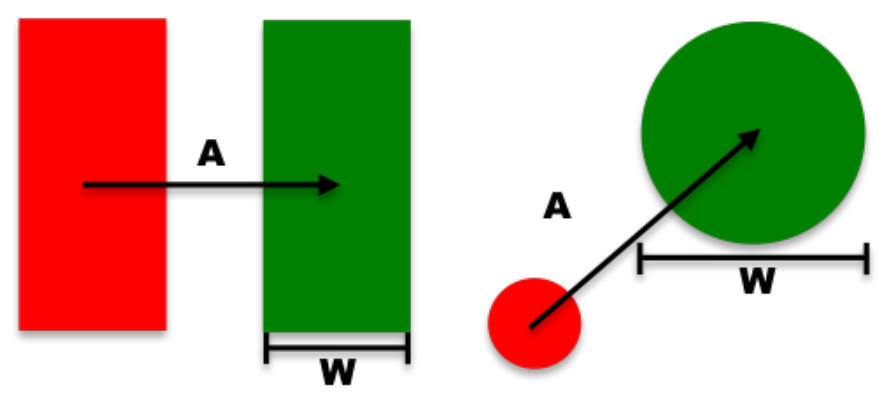

Fig. 1. The Java applet presented human subjects in our lab and online with a sequence of rectangular and circular targets and records timing data. Target distance $A$ and width $W$ remain constant (homogeneous) or vary (heterogeneous) between targets.

repetitively moved a stylus between two fixed metallic contact plates as quickly as possible for 15 seconds. Fitts measured the time $T$ required to move back and forth between the targets for 16 human volunteers, recording how timing changes with the width $W$ of the plates and the amplitude (distance) $A$ between them. Influenced by Shannon's Information Theory, Fitts empirically fitted a logarithmic model to the data yielding the now-classic "Fitts' Law".

Since 1954, many researchers have repeated these experiments under varying conditions and proposed alternate models and derivations. Most researchers focus on two-parameter models, which are intuitive for designers but do not capture all of the nuances of human velocity profiles which include higher order terms (and in many cases reversals and overshoots).

This paper also focuses on two-parameter models and presents two large datasets. Meyer et al. considered Fitts' original data and performed additional experiments with four human subjects performing wrist rotation movements to heterogeneous targets which suggest that in some cases the square-root model is superior to the logarithmic model. To explore this hypothesis, we designed and implemented a Javabased applet that can be run from any browser on the Internet. Meyer et al. proposed a Stochastic Optimized-Submovement (SOSM) derivation [3] of the Square-Root variant, summarized in section II-D. We also present a succinct derivation of the 
Square-Root variant based on optimal control theory.

The datasets and Java applet are available online at http://automation.berkeley.edu/fitts/. (Since this study was performed, Java is disabled by default on most browsers due to security concerns, but can be installed by visiting java.com) The applet presents the visitor with a sequence of visual targets to click on, records completion times, and sends the data back to our lab. We used this applet for two user studies. The first is a controlled (in-lab) study with all volunteers using the same mouse and settings. Our second study is a uncontrolled, web-based study based on an indeterminate number of volunteers who visited the website (it is possible to visit more than once) using a variety of mouse types and settings. Uncontrolled (also known as "in the wild") studies on the web do not provide the consistency of controlled inlab studies but can collect data from large numbers of diverse human participants in a variety of settings so may have greater "ecological validity" than controlled lab studies. Uncontrolled studies make it difficult to obtain reliable data about subjects and it is hard to know if they repeat the experiment multiple times. In a recent survey, Andreasen et al. [4] note that "it would be interesting to perform comparative studies of remote usability testing methods" against controlled studies.

Uncontrolled experiments are gaining acceptance in the Computer Human Interaction community. Our uncontrolled study was motivated by concerns that results might vary between a controlled lab setting and web-based settings with many different experimental environments. We were surprised to find that data from the controlled and uncontrolled studies are remarkably consistent.

\section{RELATED WORK}

There is a vast body of related work on this subject; we apologize for the inevitable errors of omission.

\section{A. Classic Fitts' Law}

In "choice reaction time tasks," a set of stimuli are assigned unique responses, and participants must give the correct response when receiving the stimulus [5]. In 1885, Merkel designed an experiment in which the stimulus was a number selected from a set with uniform probability; the participant was required to press a key corresponding to the number [5]. Merkel found that reaction time increased by a constant for every doubling of the size of the set.

In 1948, Claude Shannon published the foundational paper on Information Theory, defining the information capacity for a communication channel, $C$, as:

$$
C=B \log _{2}\left(\frac{S+N}{N}\right),
$$

where $B$ is the channel bandwidth, $S$ is signal strength, and $N$ is noise power. Shannon also defined the information $I$ of a symbol based on the probability of receiving the symbol, $I=\log _{2} \frac{1}{p}$.

Adopting Shannon's model, Merkel's reaction time can be viewed as proportional to the amount of "information" received by the participant:

$$
T_{R}=a+b \log _{2} M
$$

where $a$ and $b$ are experimentally determined constants and $M$ is the size of the set of integers.

Hick's Law models the reaction time in the cases that there are more than one target. Hick proposes a model in which the reaction time for when the person has to choose between a set of options is modeled as a logarithmic model [6] and [7]. In this model the reaction time as a logarithmic function of the number of choices $M$ is $T=\alpha+\beta \log _{2}(M)$.

In 1953, Hyman extended the work of Hick and Merkel to cases where an element $i$ in the set of possible integers was selected with non-uniform probability $p_{i}$ [8]. Hyman found that the average reaction time was also consistent with the Shannon's model [5].

In 1954, Fitts hypothesized that the information capacity of the human motor system is specified by its ability to produce consistently one class of movement from among several alternative classes of movements [2]. Fitts then defined the difficulty of a task based on the minimum amount of "information" required to complete it on average. For the "tapping task", Fitts defined a tap between two targets each of width of $W$ separated by distance (amplitude) $A$ as a movement class. Inspired by Shannon's definition of information, Fitts defined the "index of difficulty" $(I)$ based on the "information" transmitted during the task:

$$
I=\log _{2}\left(\frac{2 A}{W}\right) .
$$

Fitts noted that the choice of the numerator for this index "is arbitrary since the range of possible amplitudes must be inferred," so $2 A$ was selected rather than $A$ to ensure that the index is positive in "all practical situations."

Fitts then modeled movement time $T$ as a linear function of the "information" transmitted, producing his classic twoparameter Logarithmic model:

$$
T=a+b \log _{2}\left(\frac{2 A}{W}\right)
$$

In this paper we refer to this as the LOG model.

Although Fitts' Logarithmic model was originally developed for industrial pick-and-place tasks [2], it has been applied to a variety of human reaching movements. Card, English, and Burr at Xerox PARC applied Fitts' Logarithmic model to Human Computer Interaction and studied response times for four input devices: mouse, joystick, step keys, and text keys [9]. They found that Fitts' Logarithmic model accounts for the variation in movement time to select text on a CRT monitor using mice and joysticks. Subsequent studies applied Fitts' Logarithmic model to pen input devices [10]. Fitts' Logarithmic model has also been applied to robotics applications including telemanipulation tasks with remote video viewing [11] and pairs of participants performing tapping motions using a robot manipulator [12]. Kristensson proposes using context information, such as pattern recognition of likely key presses on a stylus keyboard, to develop input devices that increase the speed of input beyond what would be predicted by Fitts' Logarithmic model [13]. 


\section{B. Variants of Fitts' Model}

In 1960, Welford proposed a revised model based on a Weber fraction where the user must select "a distance from a total distance extending from his starting point to the far edge of the target" $[14,15]$. For some constant $b$, Welford's formulation is given by:

$$
T=b \log \left(\frac{A+\frac{1}{2} W}{W}\right)=b \log \left(\frac{A}{W}+0.5\right) .
$$

In 1992, MacKenzie developed a variation on Fitts' model that is more closely aligned with Shannon's model [16]. MacKenzie's communication channel model considers noise $N$ to be the variation around a specific signal $S$, so the signal strength equals the movement amplitude $(S=A)$ and the noise equals the width $(N=W)$. By analogy to Shannon's model (equation 1), movement time is given by:

$$
T=a+b \log _{2}\left(\frac{A}{W}+1\right) .
$$

In this paper we refer to this as the LOG' model. Other researchers such as Crossman and Welford [15] explore alternative variants of Fitts' model using communication theory.

Plamondon and Alimi review a number of studies of speed/accuracy trade-off models and their applications [17]. They categorize the experimental procedures used for the speed/accuracy trade-offs into two different categories: spatially constrained movements and temporally constrained movements. For the procedures in the first category, distance $(A)$ and the width $(W)$ are usually given and the time $(T)$ is measured. In the temporal group, movement time is given and the accuracy of reaching the target is being measured. With this definition, Fitts' Law falls into the first category. They classify different studies on the Fitts' Logarithmic model based on different types of movements (tapping, pointing, dragging), limbs and muscles groups (foot, head, hand, etc), experimental conditions (underwater, in flight, etc), device (joystick, mouse, stylus, touchpad, etc), and participants (children, monkeys, adults of different ages, etc). Hoffmann and Hui study reaching movements of fingers, wrist, forearm and shoulder. They show for the cases where an operator can choose which limb to reach a target, the limb with the smallest mass moment of inertia is often used to minimize energy needed to reach the target [18].

Other variants of Fitts' Model consider properties of the neuromuscular system, such as minimizing jerk or a sequential impulse model. Crossman and Goodeve [19] proposed a movement time model based on a sequence of discrete positional corrective motion impulses, which resulted in a Logarithmic model like Fitts' law.

Flash and Hogan show that the minimization of the jerk model is equivalent to fitting to a fifth order polynomial [20]. They developed a mathematical model of voluntary reaching movements based on maximizing the smoothness of trajectories [20]. They propose that the human motor system minimizes jerk, the derivative of acceleration. Using calculus of variations, they derive a polynomial formula for the time integral of the square of the magnitude of jerk is

$$
C=\frac{1}{2} \int_{0}^{t_{f}} \sum_{i=1}^{n}\left(\left(\frac{d^{3} x_{i}}{d t^{3}}\right)^{2}\right) d t
$$

where $n$ is the dimension of the space, $x$ is the vector coordinate of the pointer as a function of time, and $t_{f}$ is the time to reach the end point. Minimizing this formula results in 5th order polynomials with 6 unknown parameters for each dimension. One can constrain the position of the start and end points and assume the velocity and acceleration are zero at the start and end of the movement, and then solve for the parameters. The resulting trajectories have smooth position and velocity curves qualitatively similar to experimentally measured data.

Ben-Itzhak and Karniel also model arm reaching movements using jerk as a control signal and obtain minimum acceleration trajectories using bang-bang control [21]. Yazdani et al. present an alternative approach to achieving minimum jerk trajectories using a bang-bang controller that has no free parameters [22]. These jerk-based models are highly accurate when compared to recorded human movements but do not explicitly consider the effect of varying the size of a target region.

In 1988, Hoffmann and Gan proposed a model for ballistic arm movements in which the movement time is only a function of the amplitude, $T=a+b \sqrt{A}$ [23]. In 1992, Réjean Plamondon proposed an alternative to Fitts' Logarithmic model using a neuromuscular impulse response model [24, 25, 26]. Plamondon's theory for rapid human movements is based on the synergy between the agonist and antagonist neuromuscular systems [25]. In his model, the agonist and antagonist systems synchronously receive an impulse input $U_{0}\left(t-t_{0}\right)$ at time $t_{0}$ scaled by $D_{i}$, where $i=1$ for the agonist system and $i=2$ for the antagonist system. Each system independently responds in parallel to the input with impulse response functions $H_{i}(t)$ to generate output velocities $v_{i}(t)$ for $i=1,2$. Although the two systems may be coupled in reality, Plamondon assumed the output $v(t)$ of the synergy is obtained by subtracting the two parallel outputs. Plamondon proposed defining the impulse response using a log-normal function, a very general formulation based on 7 parameters that can qualitatively predict a variety of velocity profiles including single peaks, double peaks, triple peaks, asymmetric peaks, and multiple peaks with no zero crossing. Reaching movements from one point to another point terminate at a time $T$ when the velocity of motion $v(T)$ equals zero. Solving the velocity equation for the zeros using constraints set by Fitts' experiment, Plamondon modeled movement time

$$
T=K\left(\frac{2 A}{W}\right)^{\alpha}
$$

with parameters $K$ and $\alpha$.

Equation 4 defines a power model, an alternative twoparameter formulation based on a fitted log-normal approximation of the velocity profile.

Harris and Wolpert assume that the variance of the noise in the neural control signal increases with the magnitude of the control signal. They show that a smooth movement trajectory is the result of minimizing the variance of end-point position in an open-loop control mechanism [27] and [28]. Tanaka et al. (2006) extend the model proposed by Harris and Wolpert. Their model is based on optimizing movement 
time while maintaining predetermined level of accuracy [29]. More recently, Qian et al., applied an infinite-horizon optimal feedback model to goal-directed arm movements and derive both the log and power forms of Fitts' law [30].

\section{Fitts' Model for $2 D$ motions}

Fitts' Logarithmic model, which was originally developed for one-dimensional reaching movements, has been extended to the two-dimensional movements that are common in graphical user interfaces [1,31]. For general two-dimensional targets, both the shape of the target and angle of approach must be considered. For circular targets, the assumptions of the onedimensional model remain largely intact with target width $W$ being defined by the circle's diameter ${ }^{1}$. For rectangular targets, Card et al. propose a model in which the width of the target is the only contributing factor to the parameter $W$ and the height is ignored. This model can result in a negative index of difficulty for near wide targets [31, 9]. MacKenzie et al. proposed two models for rectangular targets [1]. The smaller-of model sets $W$ to the smaller of the target width or height. The effective width model sets $W$ by considering an additional parameter: the angle between the start point and the target center. MacKenzie tested the status quo, smaller-of, and effective width models and found that the linear correlation of movement time to Fitts' index of difficulty was significantly greater for both the smaller-of and effective width models compared to the status quo model [1].

Friedlander et al. found that a linear model for movement time fits selection in a non-visual (tactile or auditory) bullseye menu more closely than Fitts' Logarithmic model [32]. Gillan et al. examined how Fitts' Logarithmic model can be applied to point-drag movement sequences rather than simply pointclick operations. They found that Fitts' Logarithmic model must first be applied as the user points to the left edge of the text object and then applied separately for the dragging distance [33].

Accot et al. investigated extensions for Fitts' Logarithmic model for trajectory-based interactions, such as navigating through nested menus, drawing curves, or moving in 3D worlds [34]. They developed a "steering law" similar to Fitts' Logarithmic model except the index of difficulty for steering a pointer through a tunnel is defined by the inverse of the width of a tunnel integrated over the length of the tunnel. They applied the steering law to participants using 5 input devices (tablet, mouse, trackpoint, touchpad, and trackball), and the linear correlation of movement time to the index of difficulty for steering exceeded 0.98 [35].

Apitz et al. introduce a crossing-based interface called Cross $\mathrm{Y}^{2}$ [36]. For selecting an action in CrossY a target button is crossed instead of clicked on. This fundamentally changes both the interaction and the equations for modeling the movement since the pointing device does not necessarily need to stop on the target to click on it. Apitz et al. show that

\footnotetext{
${ }^{1}$ In this paper we use the diameter of circular targets for $W$ as suggested by MacKenzie [31].

${ }^{2}$ http://www.cs.umd.edu/hcil/crossy/
}

a crossing task is as fast as, or faster than a point-and-click task for the same index of difficulty [36].

In a related line of research, Wobbrock et al. derive a predictive model for error rates instead of mean times [37]. Error rate models have practical applications in designing text entry devices and video games [37]. Hoffmann and Drury adjust the target width $W$ by considering the width of the target, its proximity to another target and the width of the finger [38]. They show that in the case that two keys are adjacent to each other and the width of the finger pad is larger than the clearance between the two keys, $W$ can be replaced by "Available $W$ " whose value is $W_{\text {avail }}=2 S-W-F$, where $W$ is the target size, $F$ is the width of the finger pad on the device, and $S$ is the target center spacing.

The computer mouse and other pointing devices usually offer configurable parameters that adjust the mapping between movement of the device and movement of the cursor on the screen. The most common adjustment is mouse speed, a type of "control-display gain" [39]. The control-display gain scales the distance $d$ the mouse moves on the table to a distance $p$ in pixels that the cursor moves on the screen. The setting of the gain can have a significant impact on movement time to a target. Thompson et al. experimentally verified that lower gains are better for low amplitude or small target movements while higher gains are better for large amplitude or large target movements [40]. This mixed result makes it difficult to select a single optimal gain for standard computer usage. Blanch et al. introduce semantic pointing, a technique that improves target acquisition by decoupling the visual size of a target from the motor size of the target by dynamically adjusting the control-display gain when the cursor moves over a target [41]. Other device configuration parameters include acceleration and threshold [39]. When the mouse speed exceeds the threshold, the control-display gain is scaled by the acceleration parameter. Recent operating systems commonly use more complex mouse acceleration models and multiple thresholds [42].

\section{The Square-Root Variant of Fitts' Model}

Several researchers have considered a two-parameter square-root model:

$$
T=a+b \sqrt{\frac{A}{W}} .
$$

In this paper we refer to this as the SQR model. Meyer et al. proposed the Stochastic Optimized-Submovement (SOSM) derivation of the SQR model [3]. Kvalseth and Meyer et al. noted that the SQR model behaves similarly to the logarithmic model in the standard range of index of difficulty [? 3]. Meyer et al. used the homogeneous target data from the original Fitts' paper [2], and showed that the SQR model fits the original data better than the LOG model [3]. Meyer et al. also performed experiments with 4 human subjects performing wrist rotation movements to heterogenous targets with similar results.

Meyer et al. derive the time $T$ to reach the target as the sum of the average time for the primary submovement $T_{1}$ and for the corrective submovement $T_{2}$. They estimate $T$ by 
minimizing its derivative with respect to the submovements and show that when $A / W>4 / z \sqrt{2 \pi}$, the value of $\mathrm{T}$ can be approximated by the SQR function, where $z$ is the z-score such that $95 \%$ of the area under a standard Gaussian distribution $N(0,1)$ falls inside $(-z, z)$.

This derivation is based on the assumption that reaching motion can be partitioned into two or more submovements, a primary ballistic submovement and secondary corrective submovements, with near-zero velocity at the transition. The derivation is not exact and is based on four strong assumptions: 1) two submovements with a stop between them, 2) submovement endpoints have Gaussian distributions around the center point of the target, and 3) the standard deviation of each Gaussian is linearly related to the average velocity during that submovement, and 4) there are strong numerical bounds on values of $A$ and $W$ for which the approximation holds.

There are several other drawbacks to this derivation [43]. As Meyer et al. note, if the participant reaches the target in a single movement, the derivation collapses to a linear model which fits the data very poorly. The approximation requires numerical bounds on values of $A$ and $W$. Furthermore, Guiard et al. note that for a fixed positive value of $A / W$ Meyer's model approaches 1 as the number of submovements $n$ approaches infinity $[44,43]$. Meyer et al. evaluated their model with onedimensional movements using wrist rotation of a dial that can be rotated to different angular targets. In their experiments, 4 participants are presented with 12 target conditions with $A / W$ values ranging from 2.49 to 15.57 . This range of $A / W$ does not violate the assumption made for their derivation. Rioul and Guiard reconsider the Stochastic Optimized Submovement Theory of Meyer et al. and show that it implies a quasilogarithmic model, which is similar but distinct from a squareroot and cube-root law [45].

\section{A Succinct Derivation of the Square-Root (SQR) VARIANT}

In this section we present a new derivation for the SQR variant of Fitts' Law that models acceleration as (1) piecewise constant as predicted by optimal control theory for minimizing movement time, and (2) proportional to target width. The hypothesis is that wider targets may be perceived by humans as "easier" to reach and hence have a larger margin for error and allow for higher accelerations. The piecewise-constant acceleration assumption facilitates the derivation but it is a strong assumption and less consistent with empirical trajectory profiles than a piecewise-constant jerk model.

It is well-known in control theory that the optimal time for a system to reach a target is obtained by "bang-bang" control, where maximal positive acceleration is maintained for the first half of the trajectory and then switched to maximal negative acceleration for the second half $[46,5]$.

We define the halfway point (the point reached at the switching time) for a human to reach a target at distance $A$ as $x_{\text {mid }}=A / 2$. Acceleration as a function of time for bangbang control is shown in Figure 2(a), where the switching time between maximum acceleration and maximum deceleration is $s=T / 2$.

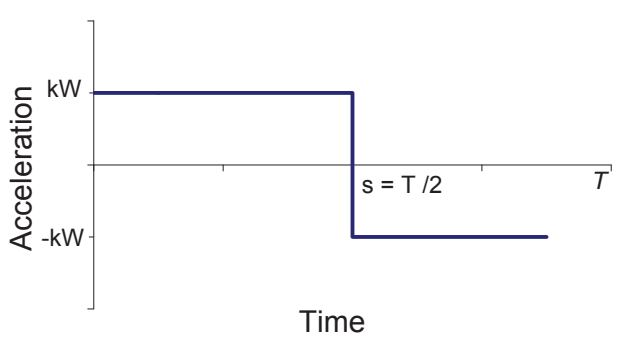

(a)

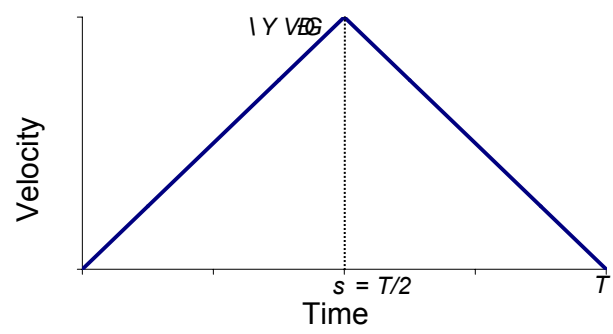

(b)

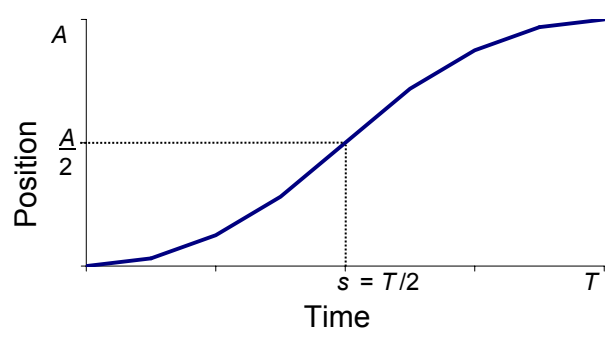

(c)

Fig. 2. Acceleration vs. Time (a), Velocity vs. Time (b), and Position vs. Time (c) under symmetric optimal control. The "bang-bang" controller maintains the maximal positive acceleration in the first half of the motion and then switches to the maximal negative acceleration until the target is reached (a). The maximal velocity is reached in the middle of the path (b).

As shown in Figure 2, Acceleration has only two values: full forward or full reverse, hence the term "bang-bang". Velocity is initially zero and then ramps up linearly during the first phase and ramps down during the second. Velocity is thus $\dot{x}(t)=\ddot{x} t$ during the acceleration phase $(t \leq s)$ and $\dot{x}(t)=$ $\ddot{x} s-\ddot{x}(t-s)$ during the deceleration phase $(t>s)$, where $\ddot{x}$ is the constant magnitude of acceleration.

We can integrate this linear velocity with respect to time to get a quadratic function for position $x(t)$. At the switching time $s$, the position by integration will be $x(s)=\frac{1}{2} \ddot{x} s^{2}$. By symmetry, position after time $T=2 s$ will be $x(T)=\ddot{x} s^{2}=$ $\frac{1}{4} \ddot{x} T^{2}$. For cursor motion, we set the total distance traveled during movement time $T$ as the amplitude $x(T)=A$. Hence, $A=\frac{1}{4} \ddot{x} T^{2}$ which implies

$$
T=2 \sqrt{\frac{A}{\ddot{x}}} .
$$




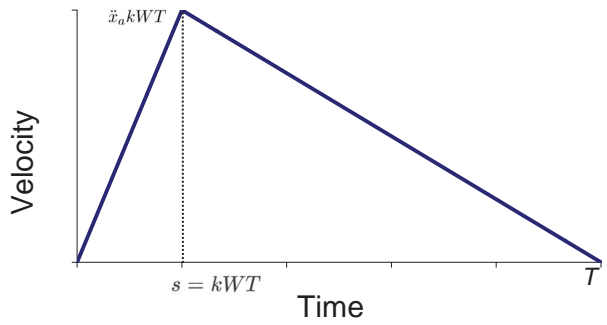

(a)

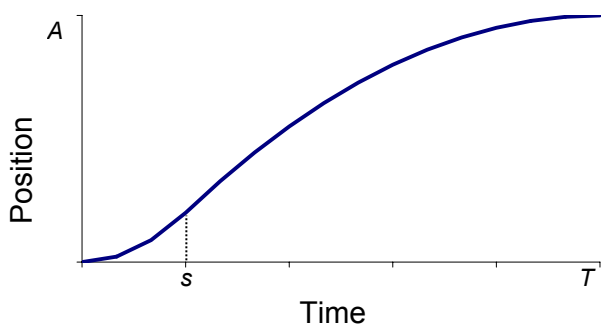

(b)

Fig. 3. Velocity vs. Time (a) and Position vs. Time (b) for the asymmetric acceleration model. Similar to MacKenzie we assume that the velocity profile is asymmetric and the peak velocity occurs at a switching time $s$ that is not necessarily equal to $T / 2$ (a) [47].

As stated above, we assume also that acceleration magnitude is proportional to the width of the target: $\ddot{x}=k W$ where $k$ is a constant scalar and $W$ is the target width. Substituting into equation 6 , we get

$$
T=2 \sqrt{\frac{A}{k W}} .
$$

We now add an initial reaction time $a$ and let $b=2 / \sqrt{k}$. The total movement time is then:

$$
T=a+b \sqrt{\frac{A}{W}} .
$$

\section{A. The SQR Variant with Asymmetric Acceleration}

In 1987, C. L. MacKenzie showed empirically that velocity profiles for reaching movements during Fitts' task are often asymmetric [47], so in this section we derive a version for asymmetric acceleration.

In this section we present a modified derivation based on an asymmetric velocity profile. Let $s$ be the switching time between the acceleration phase and deceleration phase. The peak velocity will occur at the switching time. To complete the reaching movement of amplitude $A$ with $\dot{x}(T)=0$, the magnitude of constant acceleration $\ddot{x}_{a}$ before time $s$ may be different from the constant deceleration $\ddot{x}_{d}$ after $s$.

MacKenzie showed that normalized time to peak velocity $s / T$ increases roughly linearly as target width $W$ increases and does not depend on amplitude $A$ [47]. We approximate the normalized time to peak velocity as linearly proportional to $W$ :

$$
\frac{s}{T}=k W
$$

where $k$ is a scalar constant. We also assume that initial acceleration $\ddot{x}_{a}$ for an individual is a fixed maximum acceleration regardless of the task and the deceleration $\ddot{x}_{d}$ is set so velocity is 0 at time $T$. The maximum initial acceleration condition implies $\left|\ddot{x}_{a}\right| \geq\left|\ddot{x}_{d}\right|$, consistent with MacKenzie's empirical observations [47].

To obtain a relationship between $T, A$, and $W$, we first solve for the peak velocity $\dot{x}_{\max }=\ddot{x}_{a} s$. The switching time constraint $s / T=k W$ implies $\dot{x}_{\max }=\ddot{x}_{a} k W T$. Integrating the asymmetric velocity profile in Figure 3(a) with respect to time, we get position $x(t)$, shown in Figure 3(b).

At time $T$, position as a function of $\dot{x}_{\max }$ is

$$
x(T)=\frac{1}{2} \dot{x}_{\max } s+\frac{1}{2} \dot{x}_{\max }(T-s)=\frac{1}{2} \dot{x}_{\max } T .
$$

Setting $x(T)=A$ and substituting $\dot{x}_{\max }$ into equation 8 yields:

$$
A=\frac{1}{2} \ddot{x}_{a} k W T^{2}
$$

Hence,

$$
T=\sqrt{\frac{2}{\ddot{x}_{a} k} \frac{A}{W}} .
$$

Letting $b=\sqrt{\frac{2}{\ddot{x}_{a} k}}$ and adding a fixed initial reaction time $a$ common to all trials for a given participant, we get

$$
T=a+b \sqrt{\frac{A}{W}} .
$$

Equations 9 and 7 are both binary acceleration models that were derived based on kinematic assumptions. However the assumption deriving the two equations are different, the former model assumes switching time is fixed relative to $\mathrm{T}$ and acceleration is proportional to $\mathrm{W}$ while the latter model assumes switching time is proportional to $\mathrm{W}$ and initial acceleration is a fixed constant.

\section{Controlled ANd Uncontrolled User Studies}

To compare variants of Fitts' model, we performed two user studies, one a controlled (in-lab) study and the second an uncontrolled (web-based) study. Both studies include two conditions: a "homogeneous targets" condition where sequential targets are constant in distance and size, and a "heterogeneous targets" condition where sequential targets are circular and vary in distance and size. The experiments consider targets of different difficulty, as defined by the ratio of target distance over target size as listed in Table 1 . These user studies were conducted under UC Berkeley human subject certificate IRB2009-09-283.

\section{A. The Java Applet}

For both the controlled and uncontrolled studies, we implemented a Java applet that asks each subject to complete two experiments by using his or her cursor to click on a sequence of rectangular or circular targets as they are presented on the screen. The Java applet is available online at http://automation.berkeley.edu/fitts/.

The applet records the time in milliseconds between when the target appears until the subject clicks on the target. A 
subject may click when the cursor is outside the target, but the timer increments until the target is successfully clicked upon. To facilitate precise measurement of movement times without lag from Internet communications, movement times are measured locally by the applet and sent to our central server after completion of the trials. We did not attempt to capture the complete motion trajectory since the client computer may not have sufficient processing speed when running other processes to take reliable measurements. Since the targets are measured in units of pixels, the distance and size of targets may appear different on computer systems with different display sizes and resolutions.

1) Homogeneous Targets Experiment: The Homogenous set of trials, where pairs of rectangles have the same width and are a fixed distance apart, is like the one studied in the original Fitts papers. A sequence of 33 vertical rectangles are presented as illustrated in Figure 1(a). The first, second, and third set of the 11 rectangles have the same (homogenous) width and amplitude. They hence have the same difficulty, as defined by the ratio of target distance over target size. In other words after the 11th, 22nd, and 33rd repetition, the width and amplitude (and difficulty) of the rectangles are changed. To allow subjects to "warm-up" and become familiar with each set, the system discards timing data from the first 3 timing measurements out each set of 11 , so data from the latter 8 rectangles for each difficulty is collected, producing 24 timing measurements.

2) Heterogeneous Targets Experiment: This set of trials focuses on changing targets as might be encountered in a game or computer human interface. A sequence of 25 circular targets, each of varying size and position, are presented sequentially as illustrated in Figure 1(b). Each trial begins when the subject clicks inside a small "home" circle in the center of the window and ends when the user successfully clicks inside the target. Each of the circular targets varies in distance from the home circle and varies in diameter and hence in $A / W$, difficulty level as listed in Table I.

\section{B. Two User Studies}

1) Controlled User Study Details: For the controlled user study, we posted ads on campus and Facebook offering an Amazon.com gift certificate for participation. Forty-six (46) people responded, including 17 female (37\%) and 29 male (63\%) participants. The age distribution is shown in Figure 4. The average age was 24.7 (variance $=23.8$ ). Subjects reported playing video games an average of 1.5 hours per week with variance of 10.01 hours. Out of the 46 subjects, 4 were lefthanded, but opted to use their right hand to operate the mouse. Although all of the left-handed participants were given the chance to customize their environment, none of them changed their mouse settings to left-handed; prior studies have shown that this does not disadvantage left-handed users [48].

Each participant performed the set of homogenous target and the heterogeneous target experiments in 10 trials. For this controlled experiment, we collected 490 trajectories for each of 46 subjects, giving a total of 22,540 timing measurements (11, 040 for homogenous targets and 11, 500 for heterogenous

\begin{tabular}{|c|c|c|c|c|c|c|}
\hline \multirow[t]{2}{*}{ Trial } & \multicolumn{2}{|c|}{$\begin{array}{c}\text { Homogeneous } \\
\text { Targets }\end{array}$} & \multicolumn{2}{|c|}{$\begin{array}{c}\text { Heterogeneous } \\
\text { Targets }\end{array}$} & \multirow[b]{2}{*}{$W$} & \multirow[b]{2}{*}{$A / W$} \\
\hline & $A$ & $W$ & $A / W$ & $A$ & & \\
\hline 1 & 370 & 50 & 7.40 & 67 & 20 & 3.35 \\
\hline 2 & 370 & 50 & 7.40 & 184 & 38 & 4.84 \\
\hline 3 & 370 & 50 & 7.40 & 280 & 14 & 20.00 \\
\hline 4 & 370 & 50 & 7.40 & 230 & 29 & 7.93 \\
\hline 5 & 370 & 50 & 7.40 & 144 & 55 & 2.62 \\
\hline 6 & 370 & 50 & 7.40 & 249 & 29 & 8.59 \\
\hline 7 & 370 & 50 & 7.40 & 255 & 14 & 18.21 \\
\hline 8 & 370 & 50 & 7.40 & 96 & 50 & 1.92 \\
\hline 9 & 240 & 10 & 24.00 & 225 & 19 & 11.84 \\
\hline 10 & 240 & 10 & 24.00 & 263 & 12 & 21.92 \\
\hline 11 & 240 & 10 & 24.00 & 259 & 25 & 10.36 \\
\hline 12 & 240 & 10 & 24.00 & 229 & 20 & 11.45 \\
\hline 13 & 240 & 10 & 24.00 & 215 & 31 & 6.94 \\
\hline 14 & 240 & 10 & 24.00 & 198 & 83 & 2.39 \\
\hline 15 & 240 & 10 & 24.00 & 301 & 16 & 18.81 \\
\hline 16 & 240 & 10 & 24.00 & 194 & 66 & 2.94 \\
\hline 17 & 180 & 70 & 2.57 & 260 & 12 & 21.67 \\
\hline 18 & 180 & 70 & 2.57 & 296 & 14 & 21.14 \\
\hline 19 & 180 & 70 & 2.57 & 180 & 44 & 4.09 \\
\hline 20 & 180 & 70 & 2.57 & 278 & 11 & 25.27 \\
\hline 21 & 180 & 70 & 2.57 & 283 & 37 & 7.65 \\
\hline 22 & 180 & 70 & 2.57 & 40 & 32 & 1.25 \\
\hline 23 & 180 & 70 & 2.57 & 233 & 10 & 23.30 \\
\hline 24 & 180 & 70 & 2.57 & 191 & 50 & 3.82 \\
\hline 25 & - & - & - & 179 & 18 & 9.94 \\
\hline
\end{tabular}

TARGET DiSTANCE/AMPLITUDE $(A)$ AND SIZE/WIDTH $(W)$, IN DISPLAY PIXELS, FOR THE 24 RECORDED FIXED RECTANGLES (FIXED RECTANGLES) TRIALS AND 25 VARIABLE CIRCLES TRIALS.

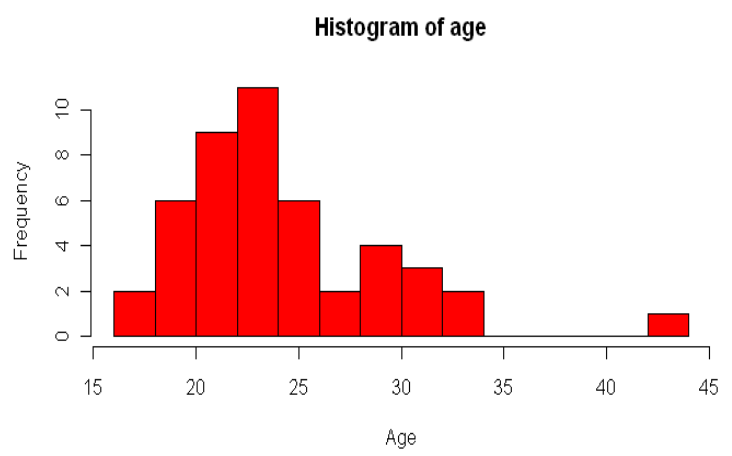

Fig. 4. Age distribution for participants for the controlled study

targets). While the order of experiments was not randomized, participants were given breaks between experiments to reduce fatigue. Allowing participants to take as many breaks as they needed was included in the experiment to make sure the final analysis does not depend on the order in which experiments were presented to participants. The experiments were performed under supervision of lab assistants who encouraged participants to repeat a trial if the participant became distracted. We cleaned the dataset by keeping only timing measurements for cases where the subject clicked on all presented targets within 3 std dev of the global mean time. This removed approximately one third of measurements where subjects did not complete the trajectory. After cleaning, the dataset contained 16,170 valid timing measurements $(8,250$ 
for homogenous targets and 7,920 for heterogenous targets).

2) Uncontrolled User Study Details: To conduct the uncontrolled study, we made the same applet available online and advertised by emails and postings on user groups. To comply with our Human Subjects approval, each online participant confirmed participation with an online consent form. We did not record IP addresses and could not determine if a person visits multiple times so we cannot determine number of unique participants. We request online visitors to indicate which type of pointing device they are using (trackpad, mouse, trackball, etc), but cannot verify the responses.

As in the controlled study, the online applet presents visitors with 24 homogenous targets and 25 heterogenous targets and thus collects up to 49 timing measurements. Unlike the controlled experiment, online visitors were not asked to repeat each experiment 10 times.

We collected timing data from 2,689 online visits to the homogeneous target experiment and 2,811 visits to the heterogenous target experiment. As in the controlled study, we cleaned the dataset by keeping only timing measurements for cases where the subject clicked on all presented targets within 3 std dev of the global mean time. This removed approximately one third of measurements where subjects did not complete the trajectory. After cleaning, the dataset included 78, 410 timing measurements, 39, 360 for homogeneous targets and 39,050 for heterogenous targets.

\section{Results}

Using root-mean-square error (RMSE), we compare three two-parameter models that relate motion duration to the ratio of target distance over target size: LOG (Fitts' original logarithmic function), SQR (square-root), and LOG' (McKenzie's logarithmic plus 1.0) [1].

We use regression to fit the unknown $a, b$ parameters for each subject and model and compute the resulting root-meansquared (RMS) error and variance. We perform two-sided paired Student t-tests comparing the within-subject models using the $p=0.05$ level of significance ${ }^{3}$. As summarized by $\mathrm{R}$. A. Fisher in his classic text, Statistical Methods for Research Workers: "The value for which $p=0.05$, or 1 in 20 , is 1.96 or nearly 2 ; it is convenient to take this point as a limit in judging whether a deviation ought to be considered significant or not. Deviations exceeding twice the standard deviation are thus formally regarded as significant."

1) Homogeneous Targets: For homogeneous targets, the results in the controlled and uncontrolled user studies are remarkably consistent. In each study, the SQR model yields significantly better fit than LOG or LOG', except for the most difficult targets.

Data from the controlled study are presented in Table II. Data from the uncontrolled study are presented in Table III. The best fit model is in the last column and is in bold face if the difference is statistically significant beyond the $p<.05$ level.

\footnotetext{
${ }^{3}$ Since the tests are repeated multiple times in this paper, a conservative Bonferroni correction can be applied to the p-value threshold to lower it to 0.0004 . This correction does not change the overall conclusion of the paper.
}

2) Heterogeneous Targets: The data are presented first using four sets of plots and then in four numerical tables (Tables 6 through 9). The plots show RMS Error and standard deviation for increasing values of difficulty for pairs of models. The first two plots compare the LOG and SQR in the Controlled and Uncontrolled Experiments respectively. The third and fourth plots compare the LOG' and SQR in the Controlled and Uncontrolled Experiments respectively. The best fit model is in the last column and is in bold face if the difference is statistically significant beyond the $p<.05$ level.

For heterogeneous targets, in both controlled and uncontrolled studies, SQR yields a significantly better fit than LOG for easier targets and LOG yields a significantly better fit for harder targets. For heterogenous targets, the LOG' model yields a better fit than LOG or SQR, except for easier targets where the results are inconclusive.

\section{Discussion AND CONCLUSION}

As summarized in the Related Work section, many models for human reaching motion have been considered in the decades since Fitts' original study. This paper focuses on twoparameter models and presents a succinct derivation of the square-root variant based on optimal control theory based on a piecewise linear model of human velocity. Human trajectories are known to contain many higher order terms (and in many cases reversals and overshoots) but we show that a linear approximation is consistent with the SQR variant proposed by Meyer et al. and performs as well or better than Fitts' LOG model for predicting measured summary statistics. Twoparameter models focus on motion duration and do not attempt to capture all of the nuances of human velocity profiles which can be very complex and usually include a number nonlinearities and asymmetrical components.

This paper presents two user studies, one a controlled (inlab) study and the second an uncontrolled (online) study. The controlled study collected 16, 170 timing measurements from 46 volunteers using the same mouse and settings. The uncontrolled (online) study collected 78,410 timing measurements from an indeterminate number of volunteers who visited the website with with a variety of mouse types and settings. Both studies include two conditions, a "homogeneous targets" condition where sequential targets are rectangular with fixed distance and size, and a "heterogeneous targets" condition where sequential targets are circular and vary in distance and size.

We use regression to fit the unknown parameters for each model and compute the resulting root-mean-squared error and variance. We perform two-sided paired Student t-tests comparing the within-subject models using the $p=0.05$ level of significance.

(1) We find that the data from the controlled and uncontrolled studies are remarkably consistent. Tables 8 and 9 exhibit inconsistency for easier targets. Uncontrolled studies are gaining acceptance and have been shown in other work to be consistent with controlled experiments $[49,50]$ as we have found in this set of studies.

(2) We find that for homogeneous targets, the SQR model yields a significantly better fit than LOG or LOG', except with 


\begin{tabular}{|c|c|c|c|c|c|c|c|c|c|c|}
\hline & \multicolumn{2}{|c|}{ SQR Model } & \multicolumn{2}{|c|}{ LOG Model } & \multicolumn{2}{|c|}{ Hypothesis Testing } & \multicolumn{2}{|c|}{ LOG' Model } & \multicolumn{2}{|c|}{ Hypothesis Testing } \\
\hline$A / W$ & $\mu_{R M S E}$ & $\sigma_{R M S E}$ & $\mu_{R M S E}$ & $\sigma_{R M S E}$ & p-value & Best Fit & $\mu_{R M S E}$ & $\sigma_{R M S E}$ & p-value & Best Fit \\
\hline 2.57 & 120.22 & 64.04 & 224.16 & 147.9 & $7.68 \mathrm{E}-28$ & SQR & 147.36 & 87.26 & $6.32 \mathrm{E}-06$ & SQR \\
\hline 7.4 & 237.92 & 132.98 & 421.8 & 291.36 & $5.26 \mathrm{E}-23$ & SQR & 299.46 & 191.16 & $2.00 \mathrm{E}-06$ & SQR \\
\hline 24 & 553.09 & 329.48 & 704.86 & 489.78 & $3.74 \mathrm{E}-06$ & SQR & 549.2 & 358.28 & $8.84 \mathrm{E}-01$ & LOG' \\
\hline
\end{tabular}

Homogeneous Targets: Controlled Study with 8,250 MeAsurements: Prediction ERror and PairwiSE Fit BETWEen SQR, LOG AND LOG' MODELS. SQR YIELDS A SIGNIFICANTLY BETTER FIT THAN LOG. IT ALSO YIELDS A SIGNIFICANTLY BETTER FIT THAN LOG' EXCEPT FOR THE MOST DIFFICULT TARGETS, WHERE THE TWO MODELS ARE NOT SIGNIFICANTLY DIFFERENT.

\begin{tabular}{|r|rr||rr|rl||rr|rr|}
\hline & \multicolumn{2}{|c||}{ SQR Model } & \multicolumn{2}{c||}{ LOG Model } & \multicolumn{2}{c||}{ Hypothesis Testing } & \multicolumn{2}{c|}{ LOG' Model } & \multicolumn{2}{c|}{ Hypothesis Testing } \\
\hline$A / W$ & $\mu_{R M S E}$ & $\sigma_{R M S E}$ & $\mu_{R M S E}$ & $\sigma_{R M S E}$ & p-value & Best Fit & $\mu_{R M S E}$ & $\sigma_{R M S E}$ & p-value & Best Fit \\
\hline 2.57 & 143.87 & 82.75 & 257.63 & 166.89 & $1.80 \mathrm{E}-120$ & SQR & 173.6 & 102.6 & $1.15 \mathrm{E}-19$ & SQR \\
\hline 7.4 & 296.63 & 177.29 & 484.39 & 322.55 & $8.84 \mathrm{E}-88$ & $\mathbf{S Q R}$ & 351.91 & 218.18 & $2.33 \mathrm{E}-15$ & SQR \\
\hline 24 & 686.34 & 423.14 & 814.39 & 545.63 & $7.68 \mathrm{E}-14$ & SQR & 649.56 & 412.45 & $1.18 \mathrm{E}-02$ & LOG' \\
\hline
\end{tabular}

Homogeneous TARgets: UnCONTRolled StUdy with 39,360 MEASUREMENTS: Prediction ERror AND PAIRWISE Fit BETWEEN SQR, LOG AND LOG' MODELS. AS IN THE CONTROLLED STUDY, SQR YIELDS A SIGNIFICANTLY BETTER FIT THAN LOG. SIMILARLY, IT YIELDS A SIGNIFICANTLY BETTER FIT THAN LOG' EXCEPT FOR THE MOST DIFFICULT TARGETS.

\section{Controlled Experiments}

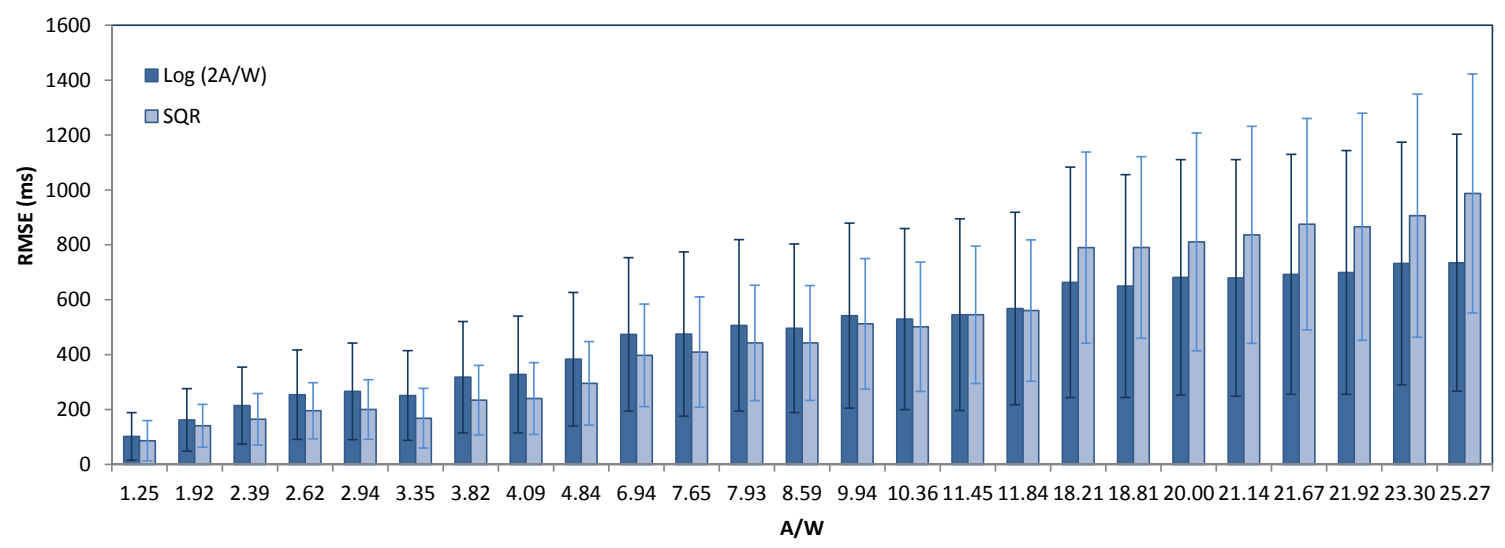

Fig. 5. Heterogeneous Targets: Controlled Study with 7,920 measurements: LOG vs SQR models. See Tables 6 through 9 for numerical details.

the most difficult targets (with higher difficulty) where the models are not significantly different. That SQR is superior is surprising in these cases since Fitts' original experiments were with homogenous targets but this is consistent with more recent experiments.

(3) We find that for heterogenous targets, SQR yields a significantly better fit than LOG for easier targets and LOG yields a significantly better fit for more difficult targets. The results are inconclusive for targets in the middle range of difficulty, while the the LOG' model yields a significantly better fit than both LOG and SQR on more difficult targets. This suggests an underlying difference in human motor processes for targets of different levels of difficulty which warrants further research.

Our applet records the time in milliseconds between when the target appears until the subject clicks on the target. We did not attempt to record the detailed motion trajectory as we were not confident that computer clients would have sufficient processing speed when running other processes to take reliable measurements, but this is an interesting direction for future work.

To our knowledge, these datasets of 94, 580 human reaching motion timing measurements are the largest collected to date. The datasets may include correlations between subsets of subjects with consistently fast response times and other structure. The anonymized datasets are freely available at: http://automation.berkeley.edu/fitts/ or available by contacting the first author.

\section{ACKNOWLEDGMENTS}

This paper is dedicated to UC Berkeley colleague Professor E.R.F.W. "Ted" Crossman, (1925-2001), who was a leading expert on time and motion studies in the Industrial Engineering and Operations Research Department. This paper is an extensively revised and expanded version of [51]. This work was supported in part by the U.S. National Science Foundation under Award IIS-1227536: Multilateral Manipulation by Human-Robot Collaborative Systems, an NSF Graduate Research Fellowship, and grants from Intel, Google, Cisco and UC Berkeley Center for Information Technology in the Interest of Society (CITRIS). We thank Jennifer Mankoff, Mikin Faldu, Amit Vijay Akula, and Anjali Koppal for their contributions to initial studies and David Rempel (UCSF), Shumin Zhai, Tamar Flash, Michael Peshkin and anonymous reviewers for helpful feedback, and all the human subjects who contributed 


\section{Uncontrolled Experiments}

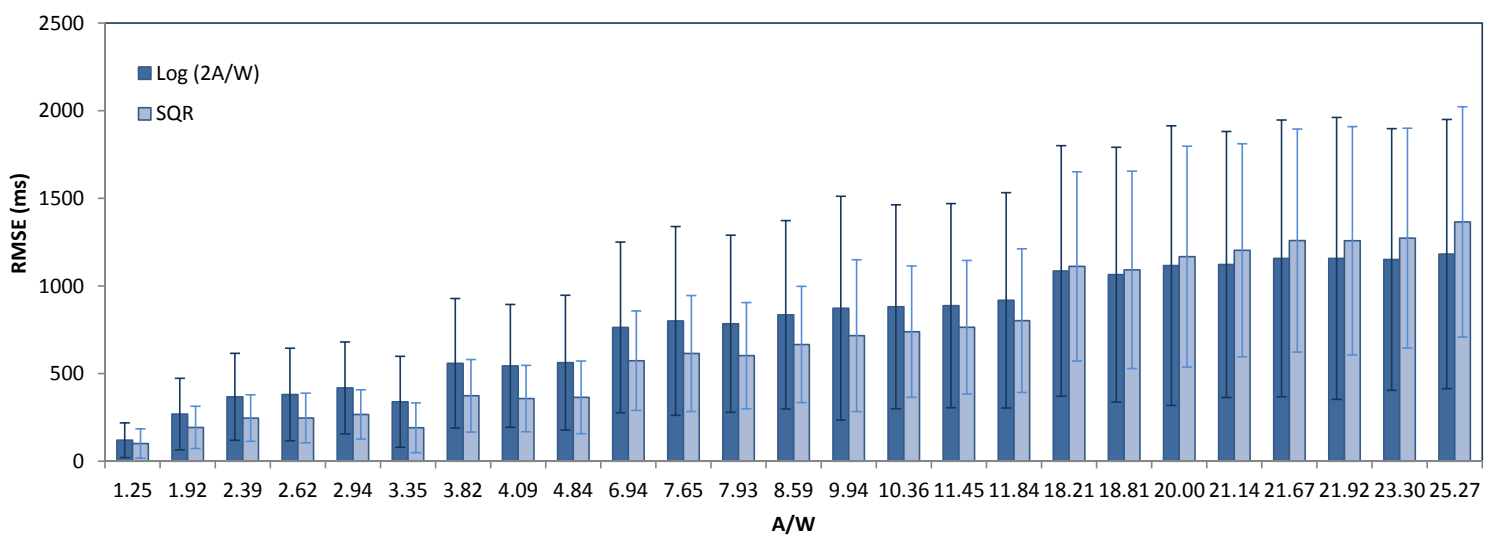

Fig. 6. Heterogeneous Targets: Uncontrolled Study with 39,050 measurements: LOG vs SQR models.

\section{Controlled Experiments}

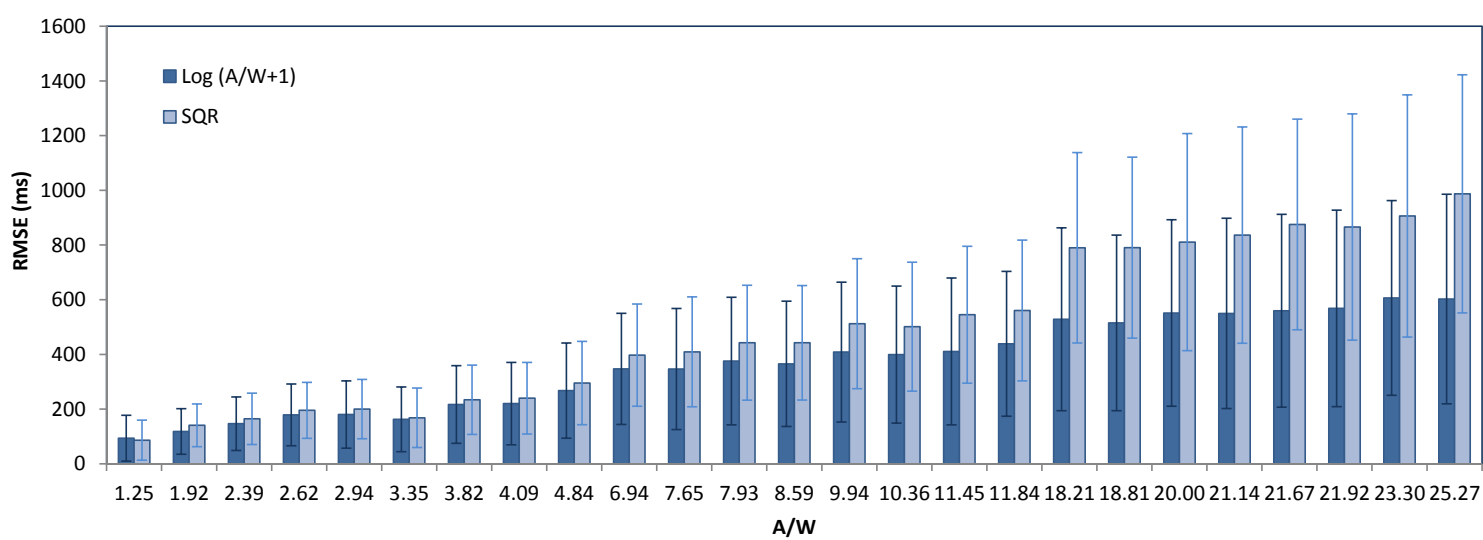

Fig. 7. Heterogeneous Targets: Controlled Study with 7,920 measurements: LOG' vs SQR models.

timing data.

\section{REFERENCES}

[1] I. S. MacKenzie and W. Buxton, "Extending Fitts' law to two-dimensional tasks," in Proc. ACM CHI '92, pp. 219-226, 1992.

[2] P. M. Fitts, "The information capacity of the human motor system in controlling the amplitude of movement," Journal of Experimental Psychology, vol. 47, pp. 381391, 1954.

[3] D. Meyer, R. Abrams, S. Kornblum, C. Wright, and J. Keith Smith, "Optimality in human motor performance: Ideal control of rapid aimed movements.," Psychological Review, vol. 95, no. 3, p. 340, 1988.

[4] M. Andreasen, H. Nielsen, S. Schrøder, and J. Stage, "What happened to remote usability testing?: an empirical study of three methods," in Proceedings of the SIGCHI conference on Human factors in computing systems, pp. 1405-1414, ACM, 2007.

[5] R. J. Jagacinski and J. M. Flach, Control Theory for Humans: Quantitative Approaches to Modeling Performance. Mahwah, New Jersey: Lawrence Erlbum Associates, 1st ed., 2003

[6] W. E. Hick, "On the rate of gain of information," Quarterly Journal of Experimental Psychology, vol. 4, no. 1, pp. 11-26, 1952

[7] M. Usher, Z. Olami, and J. L. McClelland, "Hick's law in a stochastic race model with speed-accuracy tradeoff," Journal of Mathematical Psychology, vol. 46, no. 6, pp. 704-715, 2002.

[8] R. Hyman, "Stimulus information as a determinant of reaction time," Journal of Experimental Psychology, vol. 45, pp. 188-196, 1953.

[9] S. K. Card, W. K. English, and B. J. Burr, "Evaluation of mouse, rate controlled isometric joystick, step keys, and text keys for text selection on a cathode ray tube," Ergonomics, vol. 21, pp. 601-614, 1978.

[10] I. S. MacKenzie, A. Sellen, and W. Buxton, "A comparison of input devices in elemental pointing and dragging tasks," in Proc. ACM CHI '91, pp. 161-166, 1991.
[11] D. Drascic, "Skill acquisition and task performance in teleoperation using monoscopic and stereoscopic video remote viewing," in Proc. Human Factors Society 35th Annual Meeting, pp. 1367-1371, Sept. 1991.

[12] K. Reed, M. Peshkin, J. E. Colgate, and J. Patton, "Initial studies in human-robothuman interaction: Fitts' law for two people," in Proc. IEEE Int. Conf. Robotics and Automation (ICRA), pp. 2333-2338, Apr. 2004.

[13] P.-O. Kristensson, "Breaking the laws of action in the user interface," in Proc. ACM CHI 2005, pp. 1120-1121, Apr. 2005.

[14] A. T. Welford, "The measurement of sensory-motor performance: Survey and appraisal of twelve years' progress," Ergonomics, vol. 3, pp. 189-230, 1960.

[15] A. T. Welford, Fundamentals of Skill. London: Methuen, 1968.

[16] I. S. MacKenzie, "Fitts' law as a research and design tool in human-computer interaction," Human-Computer Interaction, vol. 7, pp. 91-139, 1992.

[17] R. Plamondon and A. M. Alimi, "Speed/accuracy trade-offs in target-directed movements," Behavioral and Brain Sciences, vol. 20, pp. 279-349, 1997.

[18] E. Hoffmann and M. Hui, "Movement times of different arm components," Ergonomics, vol. 53, no. 8, pp. 979-993, 2010.

[19] E. R. F. W. Crossman and P. J. Goodeve, "Feedback control of hand-movement and Fitts' law," The Quarterly Journal of Experimental Psychology Section A, vol. 35, pp. 251-278, May 1983.

[20] T. Flash and N. Hogan, "The coordination of arm movements: An experimentally confirmed mathematical model," The Journal of Neuroscience, vol. 5, pp. 16881703, July 1985.

[21] S. Ben-Itzhak and A. Karniel, "Minimum acceleration criterion with constraints implies bang-bang control as an underlying principle for optimal trajectories of arm reaching movements," Neural Computation, vol. 20, pp. 779-812, Mar. 2008.

[22] M. Yazdani, G. Gamble, G. Henderson, and R. Hecht-Nielsen, "A simple control policy for achieving minimum jerk trajectories," Neural Networks, vol. 27, pp. 74 80, Mar. 2012.

[23] E. Hoffmann and K.-C. Gan, "Directional ballistic movement with transported mass," Ergonomics, vol. 31, no. 5, pp. 841-856, 1988.

[24] R. Plamondon, "A theory of rapid movements," in Tutorials in motor behavior II (G. Stelmach and J. Requin, eds.), pp. 55-69, New York: Elsevier Science, 1992. 


\section{Uncontrolled Experiments}

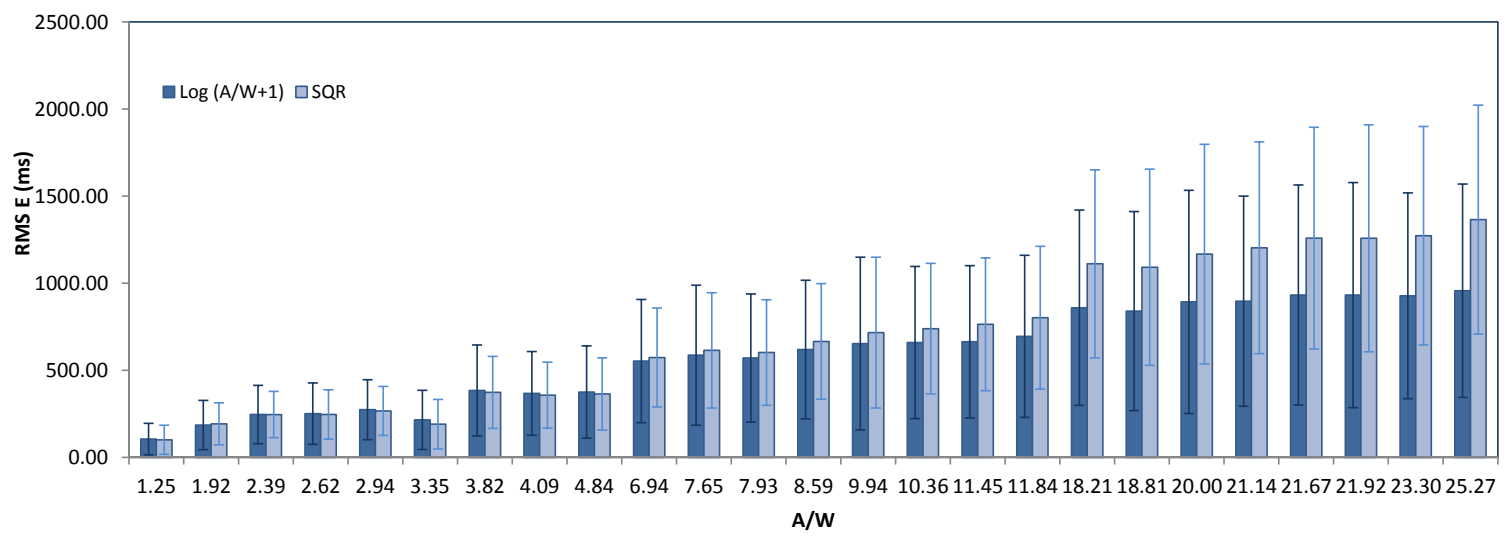

Fig. 8. Heterogeneous Targets: Uncontrolled Study with 39,050 measurements: LOG' vs SQR models.

\begin{tabular}{|c|c|c|c|c|c|c|c|c|c|c|}
\hline & \multicolumn{2}{|c|}{ SQR Model } & \multicolumn{2}{|c|}{ LOG Model } & \multicolumn{2}{|c|}{ Hypothesis Testing } & \multicolumn{2}{|c|}{ LOG' Model } & \multicolumn{2}{|c|}{ Hypothesis Testing } \\
\hline$A / W$ & $\mu_{R M S E}$ & $\sigma_{R M S E}$ & $\mu_{R M S E}$ & $\sigma_{R M S E}$ & p-value & Best Fit & $\mu_{R M S E}$ & $\sigma_{R M S E}$ & p-value & Best Fit \\
\hline 1.25 & 86.26 & 73.34 & 102.09 & 86.82 & $1.02 \mathrm{E}-02$ & SQR & 93.39 & 83.74 & $2.33 \mathrm{E}-01$ & SQR \\
\hline 1.92 & 140.68 & 78.22 & 162.18 & 113.97 & $4.42 \mathrm{E}-03$ & SQR & 118.17 & 83.18 & $4.36 \mathrm{E}-04$ & LOG' \\
\hline 2.39 & 164.37 & 93.82 & 214.24 & 140.2 & $1.38 \mathrm{E}-07$ & SQR & 146.71 & 97.94 & $1.76 \mathrm{E}-02$ & LOG' \\
\hline 2.62 & 195.47 & 102.42 & 253.96 & 162.95 & $6.79 \mathrm{E}-08$ & SQR & 178.96 & 112.99 & $4.69 \mathrm{E}-02$ & LOG' \\
\hline 2.94 & 199.92 & 108.42 & 266.23 & 176.04 & $1.28 \mathrm{E}-08$ & SQR & 180.12 & 123 & $2.71 \mathrm{E}-02$ & LOG' \\
\hline 3.35 & 168.21 & 108.85 & 250.99 & 163.39 & $1.00 \mathrm{E}-13$ & SQR & 162.56 & 118.13 & $5.20 \mathrm{E}-01$ & LOG' \\
\hline 3.82 & 234.14 & 126.64 & 317.67 & 203.14 & $4.47 \mathrm{E}-10$ & SQR & 216.83 & 142.1 & $1.06 \mathrm{E}-01$ & LOG' \\
\hline 4.09 & 239.75 & 130.97 & 327.71 & 212.77 & $4.62 \mathrm{E}-10$ & SQR & 220.04 & 150.55 & $6.95 \mathrm{E}-02$ & LOG' \\
\hline 4.84 & 295.41 & 152.44 & 383.26 & 243.54 & $4.26 \mathrm{E}-08$ & SQR & 267.82 & 174.09 & $3.36 \mathrm{E}-02$ & LOG' \\
\hline 6.94 & 397.26 & 186.73 & 473.81 & 279.8 & $4.90 \mathrm{E}-05$ & SQR & 347.05 & 203.18 & $9.34 \mathrm{E}-04$ & LOG' \\
\hline 7.65 & 409.27 & 201.03 & 474.89 & 299.14 & $1.09 \mathrm{E}-03$ & SQR & 346.52 & 221.3 & $1.54 \mathrm{E}-04$ & LOG' \\
\hline 7.93 & 442.76 & 209.92 & 506.52 & 312.39 & $2.41 \mathrm{E}-03$ & SQR & 375.59 & 233.22 & $1.11 \mathrm{E}-04$ & LOG' \\
\hline 8.59 & 442.47 & 209.2 & 495.86 & 307.24 & $8.85 \mathrm{E}-03$ & SQR & 365.48 & 228.88 & $9.72 \mathrm{E}-06$ & LOG' \\
\hline 9.94 & 512.23 & 237.91 & 541.84 & 337.11 & 2.04E-01 & SQR & 408.58 & 255.77 & $9.08 \mathrm{E}-08$ & LOG' \\
\hline 10.36 & 501.45 & 235.7 & 529.48 & 329.97 & $2.07 \mathrm{E}-01$ & SQR & 399.28 & 250.5 & $1.18 \mathrm{E}-07$ & LOG' \\
\hline 11.45 & 545.01 & 250.04 & 545.5 & 349.61 & $9.92 \mathrm{E}-01$ & SQR & 410.84 & 268.34 & $5.82 \mathrm{E}-11$ & LOG' \\
\hline 11.84 & 560.45 & 257.54 & 567.83 & 350.57 & 7.35E-01 & SQR & 438.75 & 264.69 & $5.19 \mathrm{E}-09$ & LOG' \\
\hline 18.21 & 789.96 & 348.02 & 663.13 & 419.94 & $2.55 \mathrm{E}-05$ & LOG & 528.38 & 334.25 & $2.00 \mathrm{E}-21$ & LOG' \\
\hline 18.81 & 790.24 & 330.94 & 649.96 & 405.78 & $1.86 \mathrm{E}-06$ & LOG & 515 & 321.04 & $6.00 \mathrm{E}-25$ & LOG' \\
\hline 20 & 810.65 & 397.08 & 681.42 & 428.81 & $6.07 \mathrm{E}-05$ & LOG & 551.39 & 341.18 & $2.53 \mathrm{E}-18$ & LOG' \\
\hline 21.14 & 836.16 & 395.38 & 679.44 & 431.03 & $1.41 \mathrm{E}-06$ & LOG & 549.85 & 347.95 & $1.83 \mathrm{E}-21$ & LOG' \\
\hline 21.67 & 875.12 & 385.34 & 692.45 & 437.13 & $1.74 \mathrm{E}-08$ & $\overline{\text { LOG }}$ & 559.49 & 352.79 & $8.17 \mathrm{E}-26$ & LOG' \\
\hline 21.92 & 865.82 & 413.59 & 699.3 & 444.25 & $1.08 \mathrm{E}-06$ & LOG & 568.38 & 359.3 & $3.90 \mathrm{E}-21$ & LOG' \\
\hline 23.3 & 906.26 & 442.91 & 732.01 & 442.11 & $7.10 \mathrm{E}-07$ & LOG & 606.58 & 356.09 & $3.89 \mathrm{E}-20$ & LOG' \\
\hline 25.27 & 987.07 & 435.41 & 734.77 & 468.18 & $1.83 \mathrm{E}-12$ & LOG & 602.49 & 383.22 & $2.59 \mathrm{E}-30$ & LOG' \\
\hline
\end{tabular}

HETEROGENEOUS TARGETS: CONTROLLED STUDY WITH 7,920 MEASUREMENTS: WHEN COMPARING LOG AND SQR MODELS, SQR YIELDS A SIGNIFICANTLY BETTER FIT THAN LOG FOR EASIER TARGETS AND LOG YIELDS A SIGNIFICANTLY BETTER FIT FOR HARDER TARGETS. WHEN COMPARING LOG' AND SQR MODELS, THE LOG' MODEL YIELDS A SIGNIFICANTLY BETTER FIT THAN SQR ON HARDER TARGETS (WITH HIGHER DIFFICULTY).

[25] R. Plamondon, "A kinematic theory of rapid human movements. Part I. Movement representation and generation," Biological Cybernetics, vol. 72, pp. 295-307, 1995.

[26] R. Plamondon, "A kinematic theory of rapid human movements. Part II. Movement time and control," Biological Cybernetics, vol. 72, pp. 309-320, 1995.

[27] C. M. Harris and D. M. Wolpert, "Signal-dependent noise determines motor planning," Nature, vol. 394, no. 6695, pp. 780-784, 1998.

[28] C. M. Harris and D. M. Wolpert, "The main sequence of saccades optimizes speedaccuracy trade-off," Biological cybernetics, vol. 95, no. 1, pp. 21-29, 2006.

[29] H. Tanaka, J. W. Krakauer, and N. Qian, "An optimization principle for determining movement duration," Journal of neurophysiology, vol. 95, no. 6, pp. 3875-3886, 2006.

[30] N. Qian, Y. Jiang, Z.-P. Jiang, and P. Mazzoni, "Movement duration, fitts's law, and an infinite-horizon optimal feedback control model for biological motor systems," Neural computation, vol. 25, no. 3, pp. 697-724, 2013.

[31] I. S. MacKenzie, "Movement time prediction in human-computer interfaces," in Readings in Human-Computer Interaction (R. M. Baecker, W. A. S. Buxton, J. Grudin, and S. Greenberg, eds.), pp. 483-493, Los Altos, CA: Kaufmann, 2nd ed., 1995.
[32] N. Friedlander, K. Schlueter, and M. Mantei, "Bullseye! When Fitts' law doesn't fit," in Proc. ACM CHI '98, pp. 257-264, Apr. 1998.

[33] D. J. Gillan, K. Holden, S. Adam, M. Rudisill, and L. Magee, "How does Fitts' law fit pointing and dragging?," in Proc. ACM CHI '90, pp. 227-234, Apr. 1990.

[34] J. Accot and S. Zhai, "Beyond Fitts' law: Models for trajectory-based HCI tasks," in Proc. ACM CHI '97, pp. 295-302, Mar. 1997.

[35] J. Accot and S. Zhai, "Performance evaluation of input devices in trajectory-based tasks: An application of the Steering law," in Proc. ACM CHI '99, pp. 466-472, May 1999.

[36] G. Apitz, F. Guimbretière, and S. Zhai, "Foundations for designing and evaluating user interfaces based on the crossing paradigm," ACM Trans. Comput.-Hum. Interact., vol. 17, pp. 9:1-9:42, May 2008.

[37] J. Wobbrock, E. Cutrell, S. Harada, and I. MacKenzie, "An error model for pointing based on fitts' law," in Proceedings of the twenty-sixth annual SIGCHI conference on Human factors in computing systems, pp. 1613-1622, ACM, 2008.

[38] E. Hoffmann and C. Drury, "Comment on visual layout modulates fittss law: The importance of first and last positions," Psychonomic bulletin \& review, pp. 1-5, 2011 . 


\begin{tabular}{|c|c|c|c|c|c|c|c|c|c|c|}
\hline & \multicolumn{2}{|c|}{ SQR Model } & \multicolumn{2}{|c|}{ LOG Model } & \multicolumn{2}{|c|}{ Hypothesis Testing } & \multicolumn{2}{|c|}{ LOG' Model } & \multicolumn{2}{|c|}{ Hypothesis Testing } \\
\hline$A / W$ & $\mu_{R M S E}$ & $\sigma_{R M S E}$ & $\mu_{R M S E}$ & $\sigma_{R M S E}$ & p-value & Best Fit & $\mu_{R M S E}$ & $\sigma_{R M S E}$ & $\mathrm{p}$-value & Best Fit \\
\hline 1.25 & 100.97 & 83.8 & 119.57 & 98.92 & $1.56 \mathrm{E}-08$ & SQR & 104.93 & 90.87 & $2.06 \mathrm{E}-01$ & SQR \\
\hline 1.92 & 192.5 & 120.64 & 268.83 & 204.39 & $6.22 \mathrm{E}-36$ & SQR & 185.8 & 141.69 & $1.55 \mathrm{E}-01$ & LOG' \\
\hline 2.39 & 245.9 & 133.16 & 367.02 & 248.2 & $3.02 \mathrm{E}-61$ & SQR & 245.99 & 167.69 & $9.87 \mathrm{E}-01$ & SQR \\
\hline 2.62 & 246.15 & 141.72 & 380.05 & 264.3 & $1.32 \mathrm{E}-65$ & SQR & 250.8 & 176.23 & $4.17 \mathrm{E}-01$ & SQR \\
\hline 2.94 & 266.7 & 140.79 & 417.87 & 261.97 & $4.45 \mathrm{E}-83$ & SQR & 273.89 & 172.17 & $2.02 \mathrm{E}-01$ & SQR \\
\hline 3.35 & 190.52 & 141.98 & 338.92 & 259.77 & $4.51 \mathrm{E}-81$ & SQR & 215.11 & 169.7 & $1.16 \mathrm{E}-05$ & SQR \\
\hline 3.82 & 373.06 & 206.7 & 558.07 & 369.86 & $4.52 \mathrm{E}-63$ & SQR & 384.23 & 260.92 & $1.85 \mathrm{E}-01$ & SQR \\
\hline 4.09 & 357.48 & 189.44 & 543.8 & 350.37 & $1.87 \mathrm{E}-71$ & SQR & 367.28 & 240.42 & $2.06 \mathrm{E}-01$ & SQR \\
\hline 4.84 & 364.24 & 207.13 & 562.2 & 384.6 & $2.01 \mathrm{E}-67$ & SQR & 375.31 & 264.38 & $1.93 \mathrm{E}-01$ & SQR \\
\hline 6.94 & 573.27 & 283.94 & 763.44 & 486.99 & $3.13 \mathrm{E}-39$ & SQR & 552.96 & 353.79 & 7.69E-02 & LOG' \\
\hline 7.65 & 614.45 & 330.99 & 800.26 & 538.79 & $1.97 \mathrm{E}-30$ & SQR & 586.59 & 401.56 & $3.45 \mathrm{E}-02$ & LOG' \\
\hline 7.93 & 602.04 & 303.43 & 784.19 & 505.72 & $2.40 \mathrm{E}-33$ & SQR & 570.4 & 367.8 & $8.76 \mathrm{E}-03$ & LOG' \\
\hline 8.59 & 665.74 & 331.57 & 835.37 & 537.14 & $8.00 \mathrm{E}-26$ & SQR & 618.94 & 397.74 & $3.60 \mathrm{E}-04$ & LOG' \\
\hline 9.94 & 716.32 & 433.1 & 872.83 & 638.81 & $1.62 \mathrm{E}-15$ & SQR & 653.28 & 495.88 & $1.57 \mathrm{E}-04$ & LOG' \\
\hline 10.36 & 738.77 & 374.93 & 881.19 & 582.18 & $6.56 \mathrm{E}-16$ & SQR & 659.37 & 436.75 & 5.39E-08 & LOG' \\
\hline 11.45 & 764.25 & 381.57 & 887.13 & 582.48 & $3.85 \mathrm{E}-12$ & SQR & 663.57 & 437.44 & $8.62 \mathrm{E}-12$ & LOG' \\
\hline 11.84 & 801.91 & 409.63 & 917.9 & 614.22 & $6.14 \mathrm{E}-10$ & SQR & 695.07 & 465.35 & $1.16 \mathrm{E}-11$ & LOG' \\
\hline 18.21 & 1111.37 & 539.99 & 1085.7 & 715.08 & $2.58 \mathrm{E}-01$ & LOG & 859.01 & 560.57 & $1.13 \mathrm{E}-36$ & LOG' \\
\hline 18.81 & 1091.28 & 563.31 & 1064.3 & 727.25 & $2.46 \mathrm{E}-01$ & LOG & 839.83 & 571.25 & $1.95 \mathrm{E}-34$ & LOG' \\
\hline 20 & 1167.2 & 630.27 & 1116 & 797.88 & $4.66 \mathrm{E}-02$ & LOG & 892.45 & 640.79 & $7.12 \mathrm{E}-33$ & LOG' \\
\hline 21.14 & 1203.31 & 607.69 & 1122.52 & 758.94 & $1.03 \mathrm{E}-03$ & LOG & 896.95 & 603.33 & $4.82 \mathrm{E}-44$ & LOG' \\
\hline 21.67 & 1258.6 & 636.56 & 1157.07 & 790.1 & $7.83 \mathrm{E}-05$ & LOG & 932.24 & 631.92 & $1.89 \mathrm{E}-45$ & LOG' \\
\hline 21.92 & 1258.05 & 651.53 & 1156.89 & 804.5 & $1.15 \mathrm{E}-04$ & LOG & 931.79 & 646.3 & $1.58 \mathrm{E}-43$ & LOG' \\
\hline 23.3 & 1272.71 & 627.45 & 1151.02 & 746.97 & $8.67 \mathrm{E}-07$ & LOG & 927.85 & 590.91 & $3.09 \mathrm{E}-54$ & LOG' \\
\hline 25.27 & 1364.91 & 657.32 & 1181.75 & 768.04 & $1.00 \mathrm{E}-12$ & LOG & 956.81 & 612.56 & $1.24 \mathrm{E}-68$ & LOG' \\
\hline
\end{tabular}

HeTEROGENEOUS TARGETS: UNCONTROLLED STUDY WITH 39,050 MEASUREMENTS: WHEN LOG AND SQR MODELS ARE COMPARED, AS IN THE CONTROLLED STUDY, SQR YIELDS A SIGNIFICANTLY BETTER FIT THAN LOG FOR EASIER TARGETS AND LOG YIELDS A SIGNIFICANTLY BETTER FIT FOR HARDER TARGETS. SIMILARLY, WHEN LOG' AND SQR MODELS ARE COMPARED,THE LOG' MODEL YIELDS A SIGNIFICANTLY BETTER FIT THAN SQR ON HARDER TARGETS.

[39] M. Moyle and A. Cockburn, "Analysing mouse and pen flick gestures," in Proc. SIGCHI-NZ Symposium On Computer-Human Interaction, pp. 19-24, July 1991.

[40] S. Thompson, J. Slocum, and M. Bohan, "Gain effects on angle of approach and cursor-positioning time with a mouse in consideration of Fitts' law," in Proc. Human Factors and Ergonomics Society 48th Annual Meeting, 2004.

[41] R. Blanch, Y. Guiard, and M. BeaudouinLafon, "Semantic pointing: Improving target acquisition with control-display ratio adaptation," in Proc. ACM CHI 2004, pp. 519-526, Apr. 2004.

[42] Microsoft Corporation, "SystemParametersInfo function help." Windows System Information Platform SDK, Microsoft Visual Studio .Net 2003, 2003.

[43] 1. Rioulo and Y. Guiard, "Power vs. logarithmic model of fitts' law: A mathematical analysis," "Math. Sci. hum. / Mathematics and Social Sciences", 2012. To Appear.

[44] Y. Guiard, F. Bourgeois, D. Mottet, and M. Beaudouin-Lafon, "Beyond the 10 bit barrier: Fitts' law in multi-scale electronic worlds," Proc. Interaction HommeMachine / Human-Computer Interaction (IHM-HCI 2001), People and Computers XV - Interactions without frontiers, pp. 573-588, Sept 2001.

[45] O. Rioul and Y. Guiard, "The power model of fitts' law does not encompass the logarithmic model," Electronic Notes in Discrete Mathematics, vol. 10, p. 6572, June 2013.

[46] J. Macki and A. Strauss, Introduction to optimal control theory. Springer, 1982

[47] C. L. MacKenzie, R. Marteniuk, C. Dugas, D. Liske, and B. Eickmeier, "Threedimensional movement trajectories in Fitts' task: Implications for control," The Quarterly Journal of Experimental Psychology, vol. 39A, pp. 629-647, 1987.

[48] E. Hoffmann, W. Chang, and K. Yim, "Computer mouse operation: is the lefthanded user disadvantaged?," Applied Ergonomics, vol. 28, no. 4, pp. 245-248, 1997.

[49] J. P. Bigham, C. Jayant, H. Ji, G. Little, A. Miller, R. C. Miller, R. Miller, A. Tatarowicz, B. White, S. White, and T. Yeh, "Vizwiz: nearly real-time answers to visual questions," in Proceedings of the 23nd annual ACM symposium on User interface software and technology, UIST '10, (New York, NY, USA), pp. 333-342, ACM, 2010.

[50] E. Bakshy, I. Rosenn, C. Marlow, and L. Adamic, "The role of social networks in information diffusion," in Proceedings of the 21st international conference on World Wide Web, WWW'12, (New York, NY, USA), pp. 519-528, ACM, 2012.

[51] K. Goldberg, S. Faridani, and R. Alterovitz, "A new derivation and dataset for fitts' law of human motion,” Tech. Rep. UCB/EECS-2013-171, EECS Department, University of California, Berkeley, Oct 2013. 